\title{
Development of Countenance Application Oriented on Combining ANEKA-Tri Hita Karana as A Mobile Web to Evaluate the Computer Knowledge and Morality
}

\author{
https://doi.org/10.3991/ijim.v13i12.10858 \\ Dewa Gede Hendra Divayana ${ }^{(凶)}$, I Putu Wisna Ariawan, Agus Adiarta \\ Universitas Pendidikan Ganesha, Singaraja, Indonesia \\ hendra.divayana@undiksha.ac.id
}

\begin{abstract}
The principal objective of this study was to introduce the existence on development of Countenance evaluation application that can be used to evaluate the moral and quality of students' computer knowledge by using evaluation indicators which oriented to combining between ANEKA values with the Tri Hita Karana concept. This research was developed by using the Borg \& Gall method, which focuses on the stages of creating a mobile web-based evaluation application, field trial stage, and revision stage of field trial results. The subjects involved in creating the application as much as three peoples, subjects involved in the field trial as much as 94 peoples, and subjects involved in the revision of field trial as much as three peoples. The instruments that were used in conducting the assessment on the field trial was in questionnaires form. The technique that was used to analyze the collected data from field trials results using quantitative descriptive techniques by determining the average of percentage from application quality that was compared to a standard score of application quality that refers to eleven's scale. The study results showed that the quality of the evaluation application belongs to a very good category, so it was ready to be implemented, and it is no need to be done the major revision.
\end{abstract}

Keywords-Mobile Web, Evaluation Application, Countenance, ANEKA, Tri Hita Karana.

\section{$1 \quad$ Introduction}

The positive impact of information technology progress has been felt in the field of education through computer utilization as a means of supporting the learning process as an effort to improve education quality. That matter is in principle following the opinion of Olaore in 2014 [1] which revealed that ICT becomes an essential element in education that can provide services and resources to support teaching activities, research, and public services to improve the education quality. That Olaore's opinion was also reinforced by the statement of Hernandez in 2017 [2], who stated that the ICT utilization is becoming a crucial thing is needed in education to realize the optimal learning process.

The ICT utilization in learning makes it easy for students in recognizing the new information so that it can expand their knowledge and experience. This matter is appropriate with the statement of Ziden et al. in 2011 [3] which revealed that the pres- 
ence of ICT in the learning process would increase students' understanding and knowledge in learning the material taught.

At the beginning of the computer's introduction in the education field, computers only functioned as a means of supporting learning in the form of instructional media. But, since the emergence of the industrial revolution 4.0, the computer paradigm which was only limited as instructional media had turned into an ICT-based learning model, such as e-learning, blended learning, hybrid learning, and virtual learning that allows students and teachers to be able to interact easily both in class or outside the classroom without being restricted time and space.

Even though the positive impact is very much felt cause the ICT existence in the field of education, but it does not close the gap, there are several negative impacts it causes. Some negative impacts or the problems that appear due to the ICT utilization freely and without supervision existence, those are: the behavior of students who are too free and dare to oppose the rules, abuse of internet access to searching for pornographic images or videos, students join the communities that are not clear and damaging their mental, students have dared to fight the teacher due to examples that are spread through the internet, and students are quickly affected by hoax information that exactly can plunge them. That matter similar to the statements of Mobi, Onyenanu, \& Ikwueto in 2015 [4] which stated that if ICT utilization is too excessive in learning so that ICT can take valuable learning time in meetings between teachers and students. Besides that, the ICT utilization in classroom learning hours excessively can change the learning experience into a playing experience so that it decreases achievement, and the access ease of porn sites can drop student's moral.

If those negative impacts are not anticipated early on, so it will able to drop student's moral. Therefore, moral education must steadfastly be given at school because moral as the determinant key of the formation of self-quality and one's success. That matter in principle was following the statements of Goodwin, Piazza, and Rozin in 2014 [5] which revealed that moral hold a key role in the formation of good perceptions towards someones' self.

To anticipate increasingly widespread of negative impact that is appeared from the misuse of ICT utilization in schools, especially in vocational high schools in the field of information technology which in fact often be involved with ICT, so it is necessary to conduct a comprehensive evaluation by using an evaluation tool that is able to provide appropriate recommendations so that teachers and policymakers in the school can make improvement efforts against improving the dominant aspects that need to be repaired related to the increase in knowledge and student's moral. One evaluation tool that can be used is the Countenance application that is oriented towards the ANEKA concept which be combined with the Tri Hita Karana concept, where the ANEKA concept is used for the foundation of quality determinant to student computer knowledge on the description matrix in the Countenance model, while the Tri Hita Karana concept is used as the foundation of determining the moral standards of students from judgment matrix in the Countenance model.

Based on those matters, so the purpose of this study was to find out the development of Countenance application based on the combination between ANEKA concept with the Tri Hita Karana concept as a tool for evaluating the moral and knowledge of 
IT vocational students in Bali Province in ICT learning. As for the problem statement of this study was how the development of the Countenance model evaluation application-oriented in the combination of ANEKA concept with Tri Hita Karana concept based on mobile web?

Some of the research results those were a background this study such as the research that was conducted by Agung S in 2015 [6] showed the evaluation existence of the learning process carried out by utilizing authentic assessment to assess the aspects of cognitive, affective and psychomotor from students' self. The obstacle that was found in Agung S's research was its evaluation standard had not internalized the values of local wisdom deeply.

The other research that was conducted by Savira and Tasrin in 2017 [7] showed existence on the internalization of Balinese local wisdom that was used in creating the innovation that sustainable, contextual, responsible and inclusive to realize superior quality in the field of public services. The results of Savira and Tasrin's research are similar to this study in terms of internalizing local wisdom values that are used to develop innovation in realizing the improvement of quality and achievement. The obstacles that were found in the Savira and Tasrin's research were not yet thorough in the internalization of Balinese local wisdom values in creating innovations so that they only focus on realizing the achievement of officer performance in providing service to the public, but it was not able to show an increase in moral of officers and stakeholders in public services yet.

The research that was conducted by Toharudin and Kurniawan in 2017 [8] showed the existence of teachers ability in creating evaluation model and remedial by internalizing the local cultural values so that it can measure the domain cognitive, affective and psychomotor. The obstacles that were found in the research of Toharudin and Kurniawan wasn't demonstrated the components and aspects base on the local wisdom values that were used to evaluate.

The research that was conducted by Anggraini and Kusniarti in 2016 [9] showed the realization of new models in character education, which were obtained from theater activities that were carried out at elementary schools. Besides that, also in their research, Anggraini and Kusniarti were able to show some characters that still need to be attention further development. The obstacles that were found in Anggraini and Kusniarti's research was that it wasn't able to present local cultural values that were used as a basis for realizing new model innovations in character education so that the quality of learning was not able achieved maximally both from cognitive, affective, and psychomotor dimensions.

The research result of Munteanu, Iamandi, \& Tudor in 2015 [10] showed that an evaluation model that based on the entrepreneurial character could improve the performance of tools which be used to analyze comparisons in entrepreneurship international based on cultural roles and entrepreneurship education. The obstacles were found in the Munteanu, Iamandi, \& Tudor's research was that it was not explained in detail regarding the cultural aspects that were used as evaluation standards.

Resiki, Vipriyanti, \& Sukerta's research in 2018 [11] described the implementation quality of the Medical Waste Management Program at Sanglah Hospital in Denpasar based on Tri Hita Karana was reviewed from the CIPP (Context-Input-Process- 
Product) evaluation component and constraints that were faced during the program implementation. The Resiki, Vipriyanti, \& Sukerta's research basically has similarities with this study in the matter of research approach which the form evaluative research, but the difference lies in the utilization of evaluation model that was used, where this study used the Countenance model, while in Resiki, Vipriyanti, \& Sukerta's research used the CIPP model. The obstacles that were found in Resiki, Vipriyanti, \& Sukerta's research was that it was not shown the dominant aspects that need to get priority of follow-up starting from the quality grade of the lowest to the highest level.

\section{Research Methodology}

The method that was used in the development of Countenance applications based on ANEKA-Tri Hita Karana as a quality evaluation tool the computer knowledge and morality on IT vocational students was a research and development method with the design of Borg and Gall that focuses on the stages of creating applications, field trials, and revision stages from results of field trials. At the stage of creating the application, it takes two software developers. Every form in the mobile web applications was created using the PHP programming language and MySQL database that the next step integrated into the smartphone in its implementation. At the stage of field trials, were a need as much as two education experts, two informatics experts, 30 teachers, and 60 students to do the testing against the evaluation application that had been formed. The instrument that was used to measure the level of quality of the application was in the form of questionnaires. The number of questionnaire items that was used in the field trial towards the evaluation application as much as 15 items. At the revision stage results of the field trial were required one software analyst and two software developers. The revised sections were carried out based on some input from the respondents when conducting field trials on the evaluation applications.

The technique which was used to analyze the data that had collected on the field trials used the quantitative descriptive technique by comparing the percentage result of the application quality level with a quality standard score that referred to the eleven's scale. As for the formula that was used to determine the quality level of evaluation application can be seen in equation (1) [12] while the standard score of evaluation application quality that referred to the eleven's scale can be seen in Table 1 [13].

Quality Percentage of the Application $=\frac{\sum(\text { Answer * Weight of Each Answer Choice })}{\mathrm{n} * \text { Highest Weight }} * 100 \%$

Notes: $\sum=$ total; $\mathrm{n}=$ the total number of questionnaire items 
Table 1. The Standards Score of Evaluation Application Quality by Reference to the Eleven's Scale

\begin{tabular}{|c|l|l|}
\hline Quality Level (\%) & \multicolumn{1}{|c|}{ Category } & \multicolumn{1}{|c|}{ Follow-up } \\
\hline $95-100$ & Excellent & Not revised \\
\hline $85-94$ & Very Good & Not revised \\
\hline $75-84$ & Good & Not revised \\
\hline $65-74$ & More than Enough & Not revised \\
\hline $55-64$ & Enough & Revised \\
\hline $45-54$ & Almost Enough & Revised \\
\hline $35-44$ & Less & Revised \\
\hline $25-34$ & Very Less & Revised \\
\hline $15-24$ & Bad & Revised \\
\hline $5-14$ & Very Bad & Revised \\
\hline $0-4$ & Poor & Revised \\
\hline & & \\
\hline
\end{tabular}

\section{$3 \quad$ Results and Discussion}

\subsection{Results}

The Stage of Creating Mobile Web-based Evaluation Application: At this stage was produced several forms on the Countenance evaluation application based on ANEKA-Tri Hita Karana. As for the forms intended, it can be seen in Figure 1 to Figure 10.

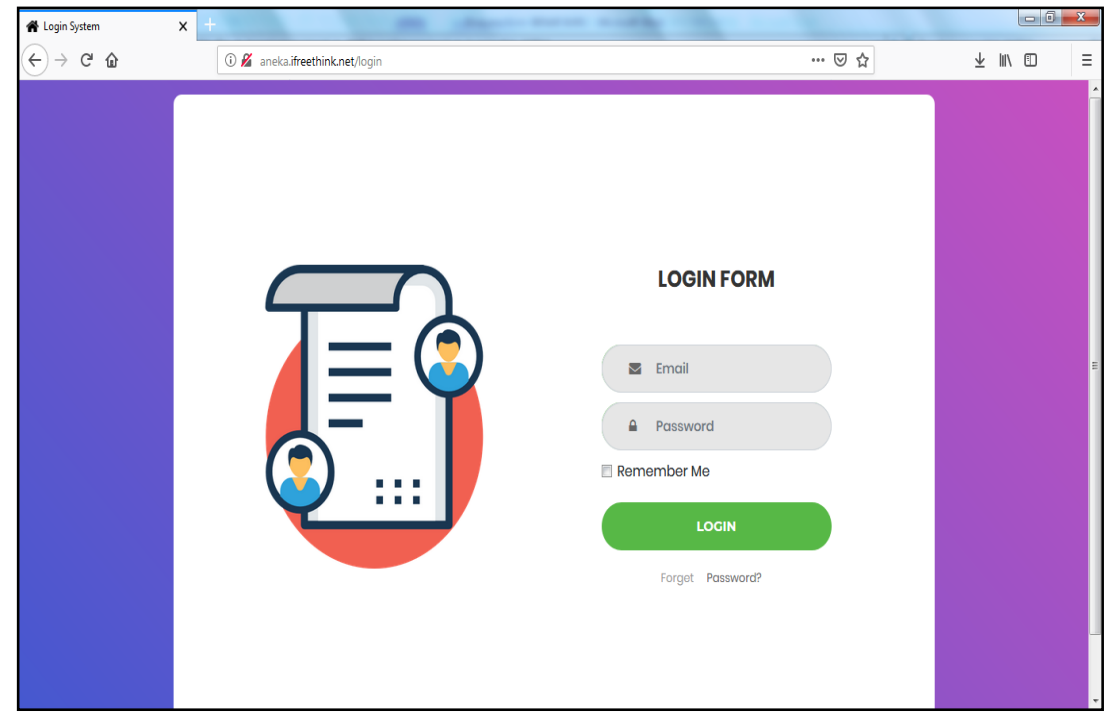

Fig. 1. Login Form 
Paper-Development of Countenance Application Oriented on Combining ANEKA-Tri Hita Karana as a..

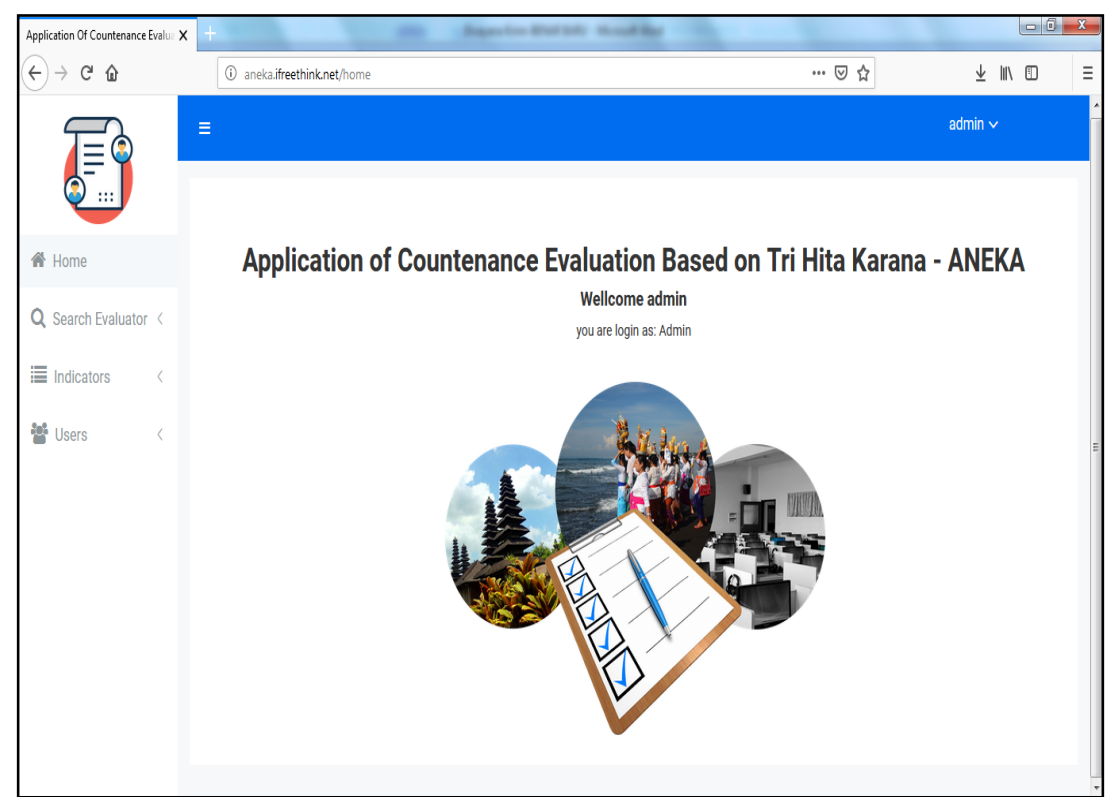

Fig. 2. Main Menu Form

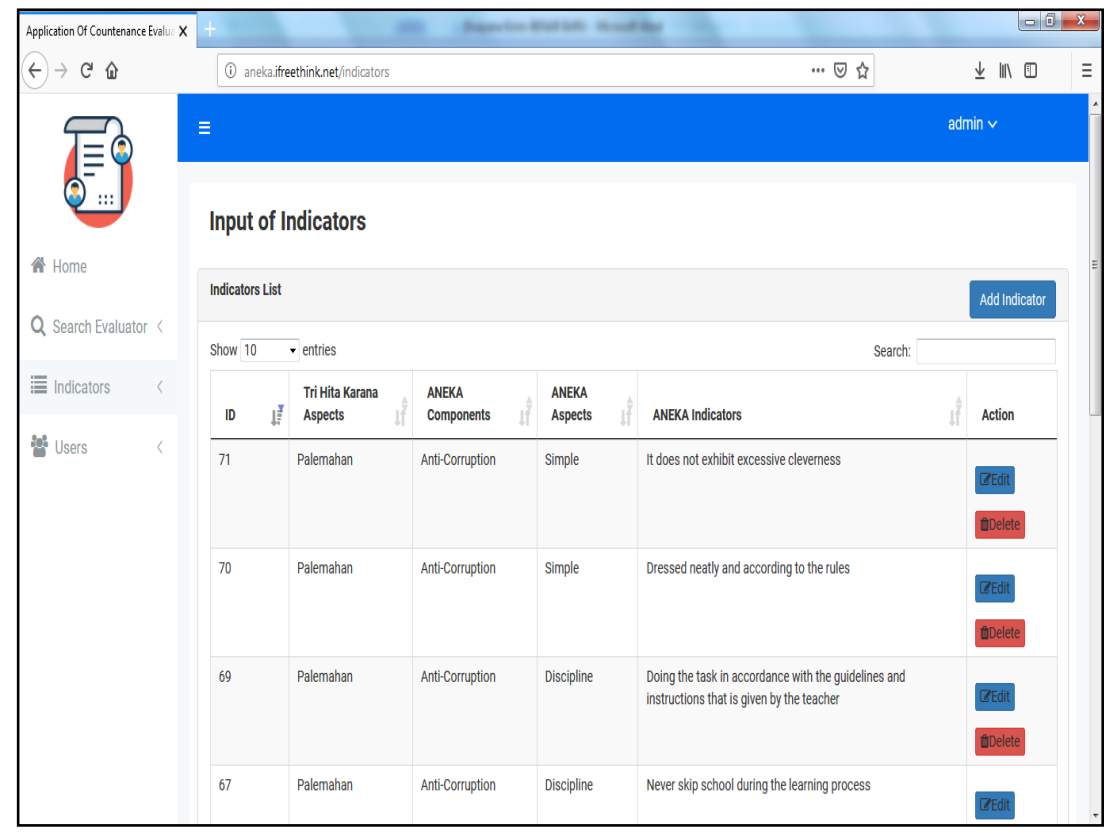

Fig. 3. Form of Indicators Input 


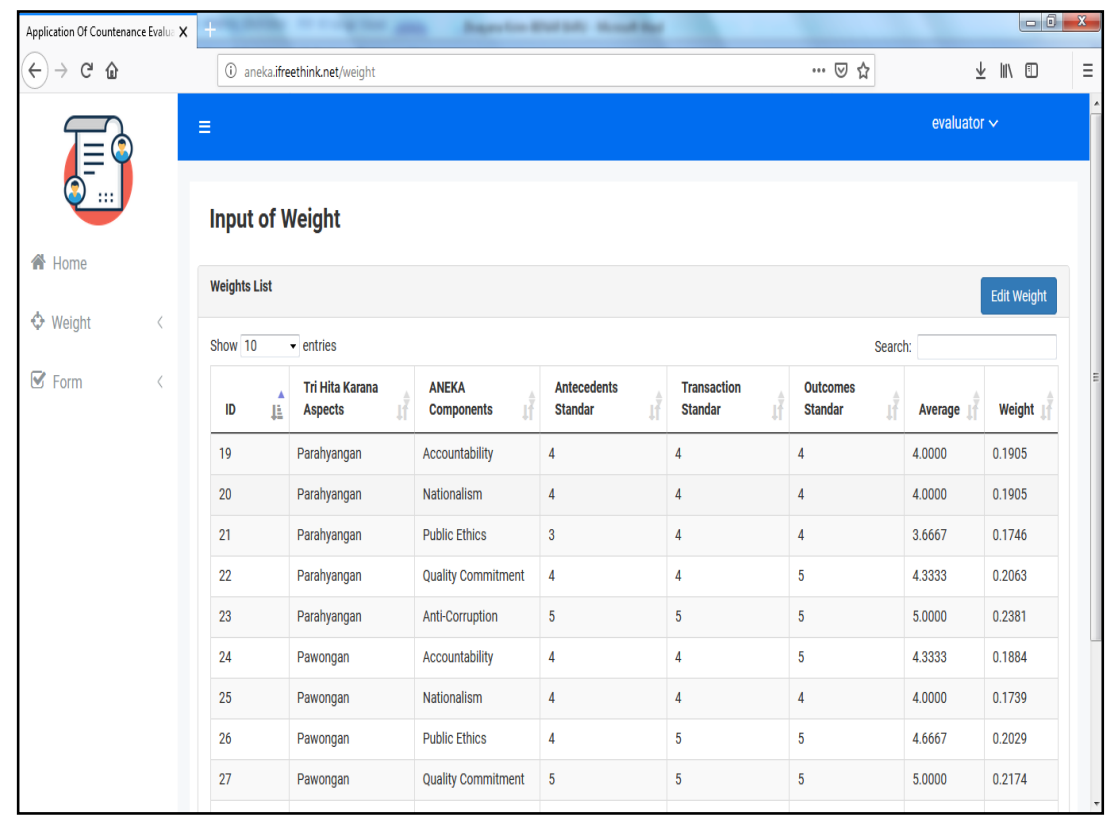

Fig. 4. Form of Weight Input

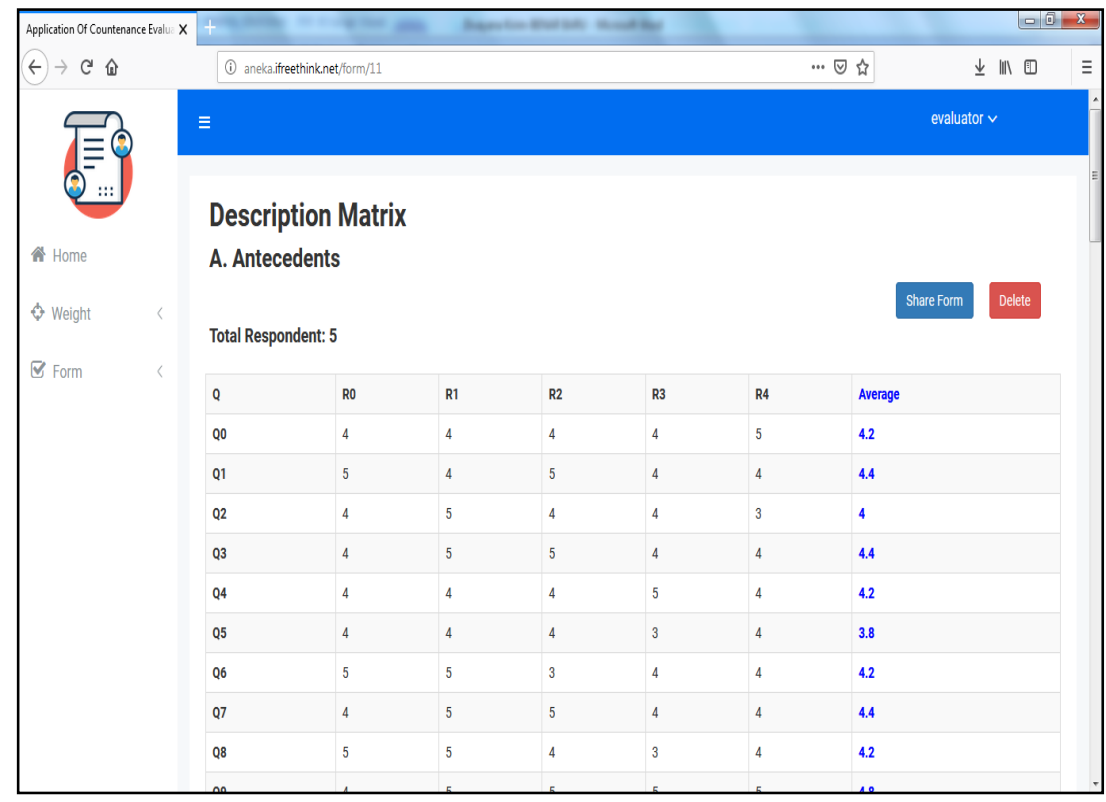

Fig. 5. Form of Antecedents on Description Matrix 


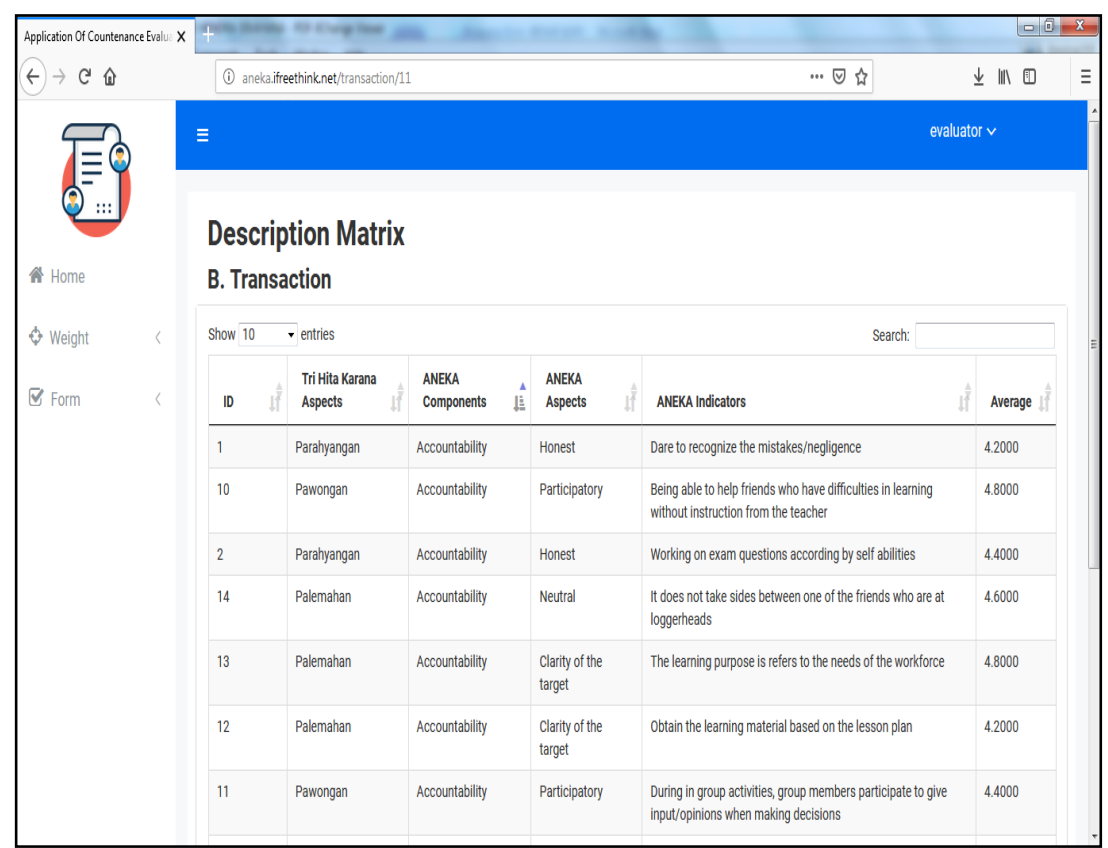

Fig. 6. Form of Transaction on Description Matrix

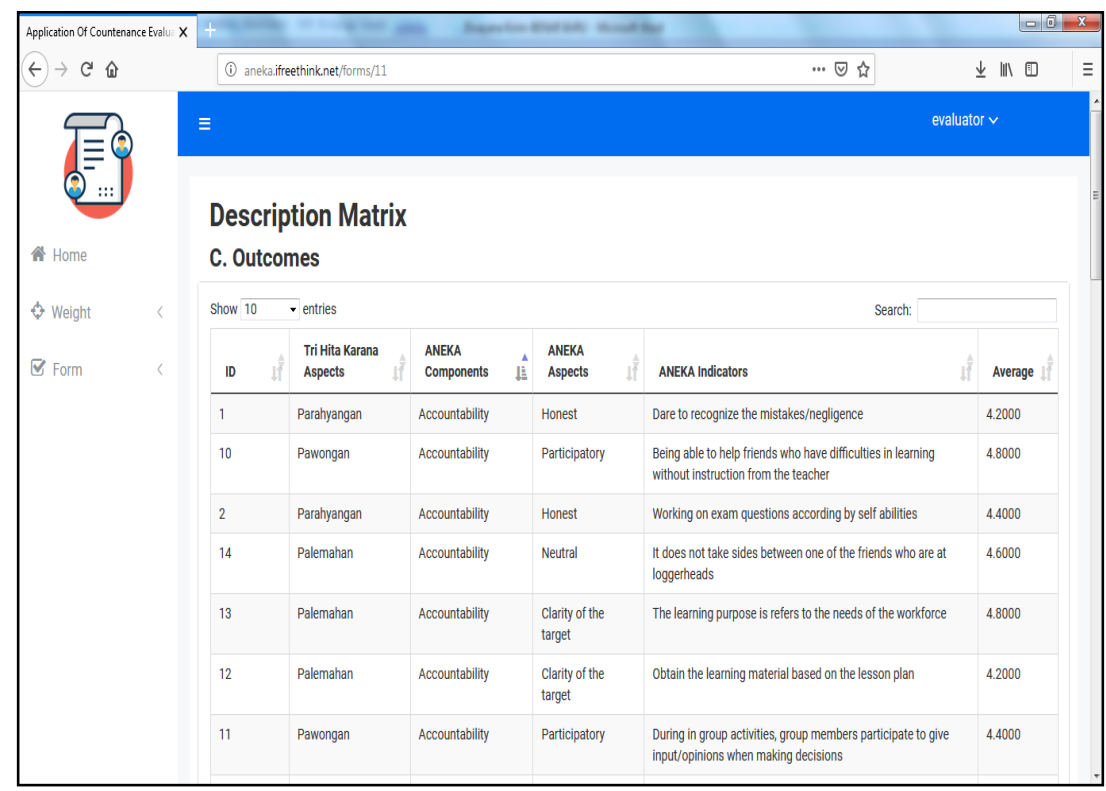

Fig. 7. Form of Outcomes on Description Matrix 


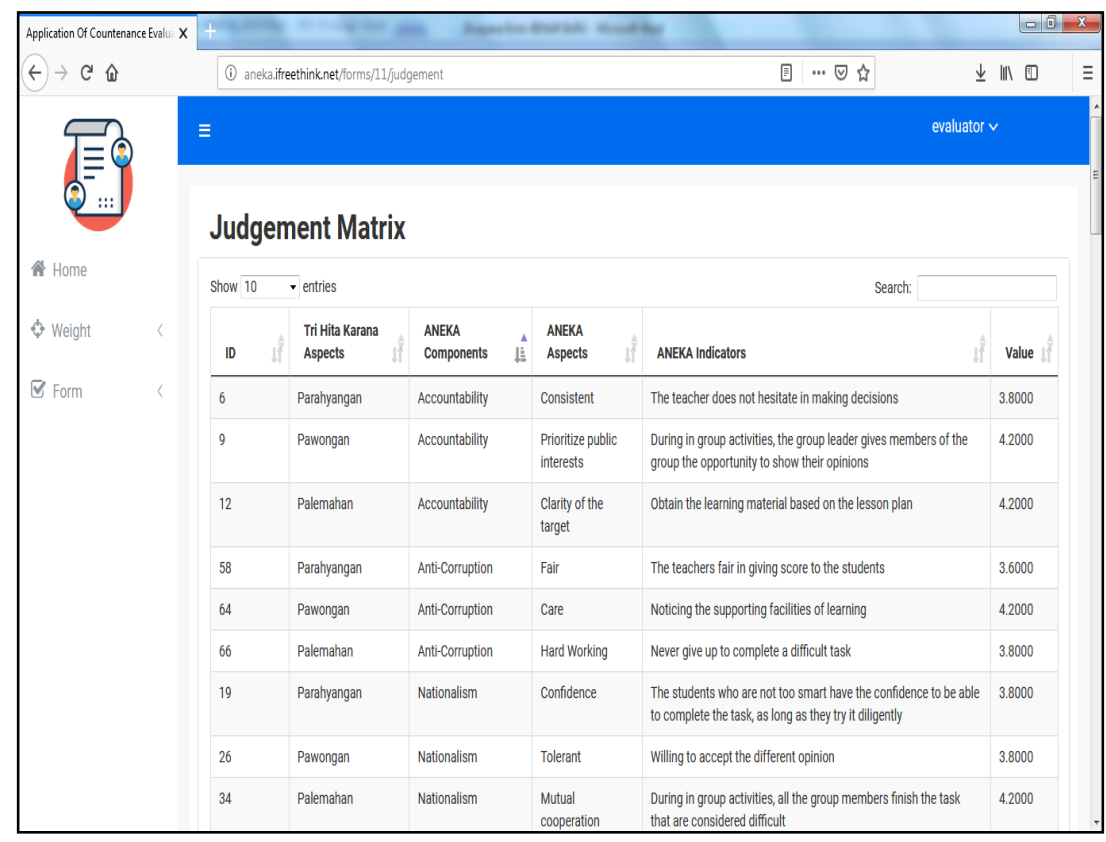

Fig. 8. Form of Judgement Matrix

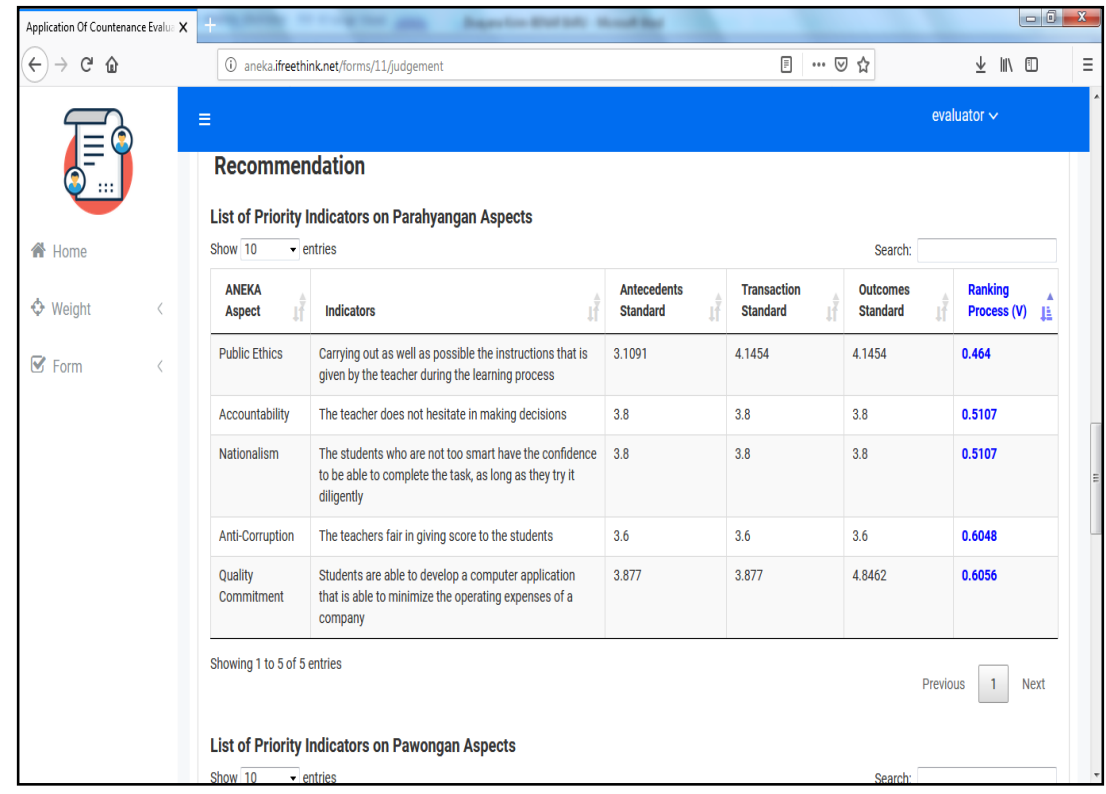

Fig. 9. Form of Recommendations 


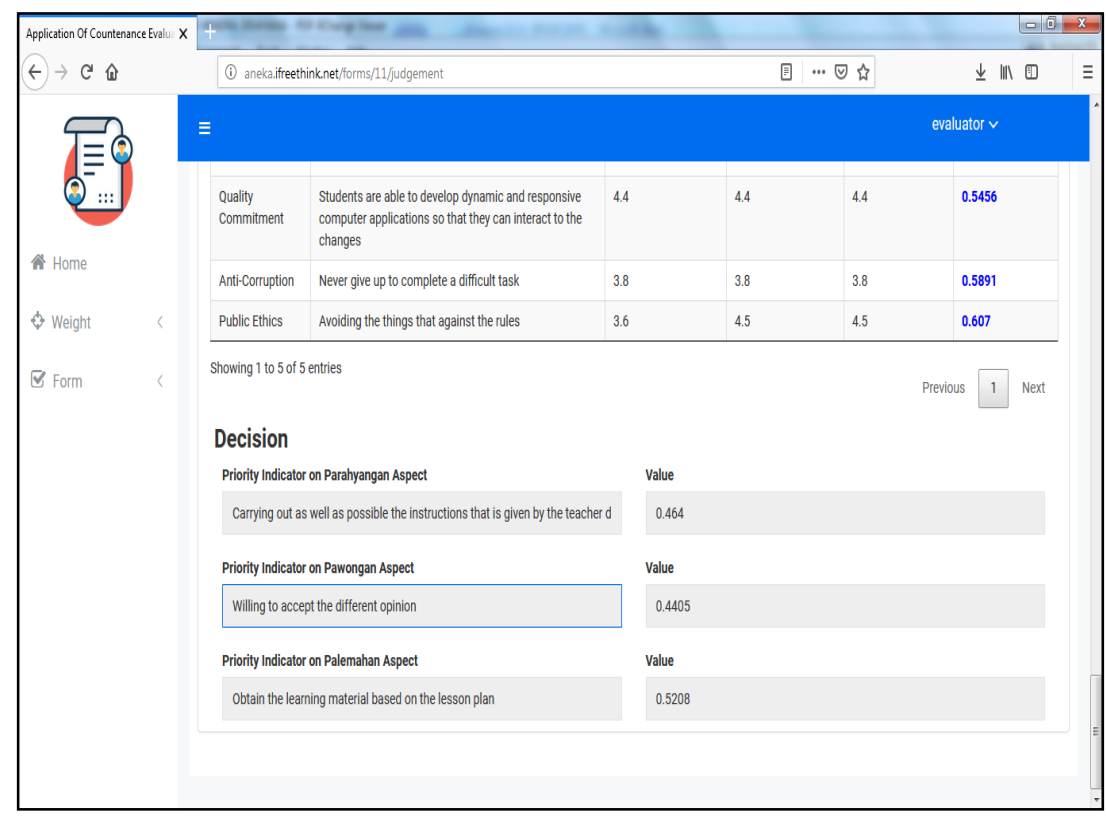

Fig. 10. Form of Decision

The Stage of Field Trial: At this stage was carried out a field trial toward Countenance evaluation application-oriented on ANEKA-Tri Hita Karana concept that involving 4 experts ( 2 education experts and 2 informatics experts), 30 teachers and 60 IT vocational students (which was spread over 6 Regencies on Bali Province), including: Tabanan, Gianyar, Buleleng, Badung, Klungkung, and Denpasar. As for the results of the field trials toward the evaluation application, it can be seen in Table 2.

Table 2. The Field Trial Results Against Countenance Evaluation Application Oriented on ANEKA-Tri Hita Karana

\begin{tabular}{|c|c|c|c|c|c|c|c|c|c|c|c|c|c|c|c|c|c|}
\hline \multirow{2}{*}{ Respondents } & \multicolumn{15}{|c|}{ Item- } & \multirow{2}{*}{$\Sigma$} & \multirow{2}{*}{$\begin{array}{c}\text { Quality } \\
\text { Percentage } \\
(\%)\end{array}$} \\
\hline & 1 & 2 & 3 & 4 & 5 & 6 & 7 & 8 & 9 & 10 & 11 & 12 & 13 & 14 & 15 & & \\
\hline Education Expert-1 & 4 & 5 & 5 & 4 & 5 & 5 & 5 & 4 & 5 & 4 & 5 & 4 & 5 & 4 & 5 & 69 & 92.00 \\
\hline Informatics Expert-1 & 5 & 4 & 4 & 4 & 4 & 5 & 4 & 4 & 5 & 5 & 5 & 4 & 5 & 4 & 4 & 66 & 88.00 \\
\hline Education Expert-2 & 5 & 5 & 5 & 4 & 4 & 5 & 4 & 4 & 4 & 4 & 4 & 5 & 4 & 5 & 4 & 66 & 88.00 \\
\hline Informatics Expert-2 & 4 & 4 & 5 & 5 & 4 & 4 & 4 & 5 & 4 & 5 & 5 & 5 & 4 & 5 & 5 & 68 & 90.67 \\
\hline Teacher-1 & 4 & 5 & 4 & 4 & 5 & 4 & 5 & 5 & 4 & 5 & 4 & 4 & 5 & 4 & 5 & 67 & 89.33 \\
\hline Teacher-2 & 4 & 4 & 4 & 5 & 4 & 5 & 4 & 4 & 5 & 4 & 5 & 5 & 4 & 5 & 4 & 66 & 88.00 \\
\hline Teacher-3 & 4 & 5 & 4 & 4 & 5 & 4 & 5 & 5 & 4 & 5 & 4 & 4 & 4 & 4 & 5 & 66 & 88.00 \\
\hline Teacher-4 & 5 & 4 & 4 & 5 & 4 & 4 & 4 & 4 & 5 & 4 & 4 & 5 & 5 & 5 & 4 & 66 & 88.00 \\
\hline Teacher-5 & 4 & 5 & 4 & 5 & 4 & 4 & 4 & 5 & 5 & 5 & 4 & 4 & 4 & 5 & 5 & 67 & 89.33 \\
\hline Teacher-6 & 5 & 5 & 4 & 4 & 4 & 5 & 5 & 4 & 5 & 4 & 5 & 5 & 4 & 4 & 5 & 68 & 90.67 \\
\hline Teacher-7 & 4 & 4 & 4 & 5 & 5 & 4 & 4 & 5 & 4 & 5 & 4 & 4 & 5 & 5 & 4 & 66 & 88.00 \\
\hline Teacher- 8 & 5 & 5 & 4 & 4 & 4 & 5 & 5 & 4 & 4 & 5 & 5 & 5 & 4 & 4 & 5 & 68 & 90.67 \\
\hline Teacher-9 & 4 & 4 & 5 & 4 & 5 & 4 & 4 & 4 & 4 & 4 & 4 & 4 & 5 & 5 & 4 & 64 & 85.33 \\
\hline
\end{tabular}




\begin{tabular}{|c|c|c|c|c|c|c|c|c|c|c|c|c|c|c|c|c|c|}
\hline \multirow[b]{2}{*}{ Respondents } & \multicolumn{15}{|c|}{ Item- } & \multirow{2}{*}{$\sum$} & \multirow{2}{*}{$\begin{array}{c}\text { Quality } \\
\text { Percentage } \\
(\%)\end{array}$} \\
\hline & 1 & 2 & 3 & 4 & 5 & 6 & 7 & 8 & 9 & 10 & 11 & 12 & 13 & 14 & 15 & & \\
\hline Teacher-10 & 5 & 5 & 4 & 4 & 5 & 5 & 5 & 5 & 4 & 5 & 4 & 5 & 4 & 5 & 4 & 69 & 92.00 \\
\hline Teacher-11 & 4 & 4 & 5 & 4 & 5 & 4 & 4 & 4 & 5 & 4 & 4 & 5 & 4 & 5 & 4 & 65 & 86.67 \\
\hline Teacher-12 & 4 & 5 & 5 & 4 & 5 & 5 & 5 & 4 & 5 & 5 & 5 & 5 & 5 & 4 & 5 & 71 & 94.67 \\
\hline Teacher-13 & 4 & 4 & 5 & 4 & 5 & 4 & 5 & 5 & 4 & 5 & 5 & 4 & 4 & 5 & 4 & 67 & 89.33 \\
\hline Teacher-14 & 4 & 5 & 4 & 4 & 5 & 4 & 4 & 5 & 4 & 5 & 4 & 4 & 4 & 4 & 5 & 65 & 86.67 \\
\hline Teacher-15 & 4 & 4 & 5 & 5 & 4 & 4 & 5 & 4 & 5 & 4 & 5 & 5 & 4 & 4 & 4 & 66 & 88.00 \\
\hline Teacher-16 & 5 & 5 & 4 & 4 & 5 & 4 & 5 & 4 & 4 & 4 & 5 & 4 & 5 & 4 & 5 & 67 & 89.33 \\
\hline Teacher-17 & 4 & 4 & 5 & 5 & 4 & 5 & 5 & 4 & 5 & 4 & 5 & 4 & 5 & 4 & 4 & 67 & 89.33 \\
\hline Teacher-18 & 5 & 4 & 5 & 4 & 5 & 4 & 5 & 4 & 4 & 5 & 5 & 4 & 4 & 5 & 4 & 67 & 89.33 \\
\hline Teacher-19 & 4 & 5 & 5 & 4 & 5 & 5 & 4 & 5 & 4 & 4 & 4 & 5 & 4 & 5 & 4 & 67 & 89.33 \\
\hline Teacher-20 & 5 & 4 & 5 & 5 & 4 & 4 & 5 & 4 & 4 & 5 & 4 & 4 & 5 & 4 & 5 & 67 & 89.33 \\
\hline Teacher-21 & 5 & 5 & 5 & 4 & 5 & 5 & 4 & 5 & 4 & 4 & 4 & 5 & 4 & 5 & 4 & 68 & 90.67 \\
\hline Teacher-22 & 5 & 4 & 4 & 4 & 5 & 4 & 5 & 4 & 5 & 5 & 4 & 4 & 5 & 4 & 5 & 67 & 89.33 \\
\hline Teacher-23 & 4 & 5 & 4 & 4 & 5 & 5 & 4 & 5 & 4 & 4 & 4 & 5 & 4 & 5 & 4 & 66 & 88.00 \\
\hline Teacher-24 & 4 & 4 & 4 & 5 & 4 & 4 & 5 & 4 & 5 & 5 & 5 & 4 & 5 & 4 & 5 & 67 & 89.33 \\
\hline Teacher-25 & 4 & 5 & 4 & 5 & 4 & 4 & 4 & 5 & 4 & 4 & 4 & 5 & 4 & 5 & 4 & 65 & 86.67 \\
\hline Teacher-26 & 4 & 4 & 4 & 5 & 5 & 4 & 5 & 5 & 5 & 4 & 5 & 5 & 4 & 4 & 4 & 67 & 89.33 \\
\hline Teacher-27 & 5 & 5 & 5 & 4 & 4 & 4 & 5 & 4 & 4 & 4 & 4 & 4 & 4 & 4 & 4 & 64 & 85.33 \\
\hline Teacher-28 & 4 & 4 & 4 & 5 & 4 & 4 & 4 & 4 & 5 & 4 & 5 & 4 & 5 & 5 & 4 & 65 & 86.67 \\
\hline Teacher-29 & 5 & 5 & 5 & 4 & 4 & 5 & 4 & 4 & 5 & 5 & 4 & 5 & 4 & 5 & 4 & 68 & 90.67 \\
\hline Teacher-30 & 4 & 4 & 4 & 5 & 4 & 4 & 5 & 4 & 5 & 5 & 4 & 5 & 4 & 5 & 5 & 67 & 89.33 \\
\hline Student-1 & 5 & 5 & 5 & 4 & 4 & 5 & 4 & 5 & 5 & 4 & 4 & 4 & 4 & 5 & 4 & 67 & 89.33 \\
\hline \begin{tabular}{|l|} 
Student-2 \\
\end{tabular} & 4 & 4 & 4 & 4 & 5 & 4 & 5 & 4 & 4 & 5 & 5 & 4 & 5 & 4 & 5 & 66 & 88.00 \\
\hline \begin{tabular}{|l|} 
Student-3 \\
\end{tabular} & 5 & 5 & 4 & 5 & 4 & 5 & 4 & 4 & 5 & 4 & 5 & 5 & 4 & 5 & 5 & 69 & 92.00 \\
\hline \begin{tabular}{|l|} 
Student-4 \\
\end{tabular} & 4 & 4 & 5 & 5 & 4 & 4 & 5 & 5 & 4 & 4 & 4 & 4 & 5 & 4 & 4 & 65 & 86.67 \\
\hline Student-5 & 5 & 5 & 5 & 4 & 5 & 5 & 5 & 4 & 5 & 5 & 4 & 4 & 4 & 5 & 5 & 70 & 93.33 \\
\hline Student- 6 & 4 & 4 & 5 & 5 & 5 & 4 & 4 & 4 & 5 & 4 & 5 & 4 & 5 & 5 & 4 & 67 & 89.33 \\
\hline \begin{tabular}{|l|} 
Student-7 \\
\end{tabular} & 5 & 5 & 4 & 4 & 5 & 4 & 5 & 4 & 5 & 4 & 4 & 5 & 5 & 4 & 5 & 68 & 90.67 \\
\hline Student-8 & 5 & 5 & 5 & 4 & 4 & 4 & 5 & 4 & 4 & 4 & 5 & 4 & 4 & 4 & 4 & 65 & 86.67 \\
\hline Student-9 & 4 & 4 & 4 & 5 & 4 & 5 & 4 & 4 & 5 & 4 & 4 & 5 & 5 & 5 & 5 & 67 & 89.30 \\
\hline Student-10 & 5 & 4 & 5 & 5 & 4 & 4 & 5 & 4 & 5 & 4 & 5 & 4 & 5 & 4 & 4 & 67 & 89.33 \\
\hline Student-11 & 4 & 4 & 4 & 4 & 5 & 5 & 4 & 5 & 4 & 5 & 5 & 5 & 4 & 5 & 5 & 68 & 90.67 \\
\hline Student-12 & 4 & 4 & 5 & 5 & 4 & 4 & 5 & 4 & 5 & 4 & 4 & 4 & 4 & 4 & 4 & 64 & 85.33 \\
\hline Student-13 & 5 & 5 & 4 & 5 & 4 & 5 & 4 & 5 & 4 & 5 & 4 & 5 & 5 & 5 & 5 & 70 & 93.33 \\
\hline \begin{tabular}{|l|} 
Student-14 \\
\end{tabular} & 4 & 4 & 5 & 4 & 5 & 4 & 5 & 4 & 4 & 4 & 4 & 4 & 4 & 4 & 5 & 64 & 85.33 \\
\hline Student-15 & 5 & 5 & 4 & 4 & 4 & 5 & 4 & 5 & 5 & 5 & 4 & 5 & 4 & 5 & 5 & 69 & 92.00 \\
\hline Student-16 & 4 & 4 & 5 & 5 & 4 & 4 & 5 & 4 & 4 & 5 & 5 & 5 & 4 & 4 & 4 & 66 & 88.00 \\
\hline Student-17 & 5 & 5 & 5 & 4 & 4 & 5 & 4 & 5 & 4 & 5 & 4 & 4 & 4 & 5 & 5 & 68 & 90.67 \\
\hline \begin{tabular}{|l|} 
Student-18 \\
\end{tabular} & 4 & 4 & 4 & 5 & 5 & 5 & 5 & 4 & 4 & 4 & 4 & 5 & 5 & 4 & 4 & 66 & 88.00 \\
\hline \begin{tabular}{|l|} 
Student-19 \\
\end{tabular} & 4 & 5 & 5 & 5 & 4 & 5 & 4 & 5 & 4 & 5 & 4 & 4 & 4 & 5 & 5 & 68 & 90.67 \\
\hline Student-20 & 5 & 4 & 4 & 5 & 5 & 4 & 5 & 4 & 5 & 4 & 4 & 5 & 5 & 4 & 4 & 67 & 89.33 \\
\hline Student-21 & 5 & 5 & 4 & 5 & 4 & 5 & 4 & 4 & 4 & 5 & 4 & 4 & 4 & 5 & 5 & 67 & 89.33 \\
\hline Student-22 & 5 & 4 & 4 & 5 & 4 & 4 & 4 & 5 & 5 & 4 & 5 & 5 & 5 & 4 & 4 & 67 & 89.33 \\
\hline Student-23 & 4 & 5 & 4 & 4 & 4 & 4 & 5 & 4 & 5 & 5 & 4 & 5 & 4 & 5 & 5 & 67 & 89.33 \\
\hline \begin{tabular}{|l|} 
Student-24 \\
\end{tabular} & 5 & 5 & 4 & 5 & 4 & 5 & 4 & 5 & 4 & 4 & 5 & 5 & 5 & 5 & 4 & 69 & 92,00 \\
\hline \begin{tabular}{|l|} 
Student-25 \\
\end{tabular} & 4 & 4 & 5 & 5 & 4 & 4 & 4 & 4 & 5 & 4 & 4 & 4 & 4 & 4 & 5 & 64 & 85.33 \\
\hline
\end{tabular}




\begin{tabular}{|c|c|c|c|c|c|c|c|c|c|c|c|c|c|c|c|c|c|}
\hline \multirow[b]{2}{*}{ Respondents } & \multicolumn{15}{|c|}{ Item- } & \multirow[b]{2}{*}{$\Sigma$} & \multirow{2}{*}{\begin{tabular}{|c} 
Quality \\
Percentage \\
$(\%)$
\end{tabular}} \\
\hline & 1 & 2 & 3 & 4 & 5 & 6 & 7 & 8 & 9 & 10 & 11 & 12 & 13 & 14 & 15 & & \\
\hline Student-26 & 5 & 4 & 4 & 5 & 5 & 5 & 4 & 5 & 4 & 5 & 5 & 5 & 5 & 5 & 4 & 70 & 93.33 \\
\hline \begin{tabular}{|l|} 
Student-27 \\
\end{tabular} & 4 & 4 & 5 & 4 & 5 & 4 & 4 & 4 & 4 & 5 & 4 & 4 & 4 & 5 & 5 & 65 & 86.67 \\
\hline Student-28 & 5 & 4 & 4 & 5 & 4 & 5 & 4 & 5 & 4 & 4 & 5 & 5 & 5 & 4 & 4 & 67 & 89.33 \\
\hline \begin{tabular}{|l|} 
Student-29 \\
\end{tabular} & 4 & 5 & 5 & 4 & 5 & 4 & 5 & 4 & 5 & 5 & 4 & 4 & 4 & 4 & 4 & 66 & 88.00 \\
\hline Student-30 & 5 & 5 & 4 & 4 & 4 & 4 & 5 & 5 & 5 & 4 & 4 & 4 & 5 & 4 & 5 & 67 & 89.33 \\
\hline Student-31 & 4 & 4 & 5 & 4 & 4 & 5 & 4 & 5 & 4 & 4 & 5 & 5 & 4 & 5 & 4 & 66 & 88.00 \\
\hline Student-32 & 5 & 4 & 4 & 4 & 5 & 4 & 5 & 4 & 5 & 4 & 4 & 4 & 5 & 4 & 4 & 65 & 86.67 \\
\hline Student-33 & 4 & 5 & 4 & 4 & 5 & 4 & 5 & 5 & 4 & 4 & 5 & 5 & 5 & 4 & 4 & 67 & 89.33 \\
\hline \begin{tabular}{|l|} 
Student-34 \\
\end{tabular} & 5 & 4 & 5 & 4 & 5 & 5 & 4 & 4 & 5 & 4 & 5 & 4 & 4 & 5 & 5 & 68 & 90.67 \\
\hline Student-35 & 4 & 5 & 5 & 4 & 4 & 4 & 5 & 4 & 5 & 5 & 4 & 4 & 5 & 4 & 4 & 66 & 88.00 \\
\hline \begin{tabular}{|l|} 
Student-36 \\
\end{tabular} & 5 & 4 & 4 & 5 & 4 & 4 & 5 & 4 & 4 & 5 & 4 & 5 & 5 & 5 & 4 & 67 & 89.33 \\
\hline \begin{tabular}{|l|} 
Student-37 \\
\end{tabular} & 4 & 5 & 4 & 4 & 4 & 4 & 5 & 4 & 5 & 5 & 5 & 5 & 5 & 4 & 4 & 67 & 89.33 \\
\hline \begin{tabular}{|l|} 
Student-38 \\
\end{tabular} & 5 & 5 & 5 & 4 & 4 & 4 & 5 & 4 & 4 & 5 & 4 & 4 & 5 & 5 & 4 & 67 & 89.33 \\
\hline \begin{tabular}{|l|} 
Student-39 \\
\end{tabular} & 4 & 4 & 4 & 5 & 5 & 4 & 4 & 4 & 5 & 4 & 5 & 5 & 5 & 4 & 5 & 67 & 89.33 \\
\hline Student-40 & 5 & 4 & 5 & 4 & 4 & 5 & 5 & 4 & 4 & 5 & 4 & 4 & 5 & 5 & 5 & 68 & 90.67 \\
\hline Student-41 & 4 & 5 & 4 & 5 & 5 & 4 & 4 & 5 & 5 & 4 & 4 & 5 & 4 & 4 & 4 & 66 & 88.00 \\
\hline Student-42 & 4 & 5 & 4 & 5 & 4 & 5 & 5 & 4 & 4 & 5 & 4 & 4 & 5 & 5 & 4 & 67 & 89.33 \\
\hline \begin{tabular}{|l|} 
Student- 43 \\
\end{tabular} & 4 & 4 & 5 & 5 & 4 & 4 & 4 & 4 & 4 & 4 & 4 & 4 & 4 & 4 & 5 & 63 & 84.00 \\
\hline \begin{tabular}{|l|} 
Student- 44 \\
\end{tabular} & 5 & 4 & 5 & 4 & 4 & 5 & 4 & 5 & 4 & 5 & 5 & 4 & 5 & 5 & 4 & 68 & 90.67 \\
\hline Student-45 & 5 & 5 & 4 & 5 & 4 & 4 & 4 & 4 & 4 & 4 & 4 & 4 & 5 & 4 & 5 & 65 & 86.67 \\
\hline \begin{tabular}{|l|} 
Student-46 \\
\end{tabular} & 5 & 4 & 4 & 4 & 4 & 4 & 4 & 4 & 4 & 5 & 5 & 4 & 4 & 5 & 4 & 64 & 85.33 \\
\hline \begin{tabular}{|l|} 
Student-47 \\
\end{tabular} & 4 & 4 & 4 & 5 & 5 & 5 & 4 & 5 & 5 & 4 & 4 & 5 & 4 & 5 & 5 & 68 & 90.67 \\
\hline Student-48 & 5 & 4 & 5 & 4 & 5 & 4 & 4 & 4 & 4 & 5 & 5 & 4 & 4 & 4 & 4 & 65 & 86.67 \\
\hline Student-49 & 4 & 5 & 4 & 4 & 4 & 5 & 5 & 5 & 5 & 4 & 4 & 5 & 4 & 5 & 5 & 68 & 90.67 \\
\hline \begin{tabular}{|l|} 
Student-50 \\
\end{tabular} & 5 & 4 & 5 & 4 & 5 & 4 & 4 & 4 & 4 & 4 & 5 & 4 & 4 & 4 & 4 & 64 & 85.33 \\
\hline Student-51 & 4 & 5 & 4 & 4 & 4 & 5 & 5 & 4 & 5 & 4 & 4 & 5 & 5 & 5 & 5 & 68 & 90.67 \\
\hline Student-52 & 5 & 4 & 4 & 5 & 4 & 4 & 5 & 4 & 4 & 4 & 5 & 4 & 4 & 4 & 4 & 64 & 85.33 \\
\hline Student-53 & 4 & 5 & 4 & 4 & 4 & 5 & 4 & 4 & 5 & 5 & 4 & 5 & 5 & 4 & 5 & 67 & 89.33 \\
\hline Student-54 & 5 & 5 & 5 & 4 & 5 & 4 & 4 & 5 & 5 & 4 & 5 & 4 & 4 & 4 & 4 & 67 & 89.33 \\
\hline Student-55 & 4 & 5 & 4 & 5 & 5 & 5 & 4 & 4 & 5 & 5 & 4 & 5 & 5 & 4 & 4 & 68 & 90.67 \\
\hline \begin{tabular}{|l|} 
Student-56 \\
\end{tabular} & 5 & 5 & 5 & 4 & 5 & 4 & 4 & 5 & 4 & 4 & 5 & 4 & 4 & 4 & 5 & 67 & 89.33 \\
\hline \begin{tabular}{|l|} 
Student-57 \\
\end{tabular} & 4 & 5 & 4 & 4 & 4 & 5 & 4 & 4 & 4 & 5 & 4 & 5 & 5 & 4 & 4 & 65 & 86.67 \\
\hline \begin{tabular}{|l|} 
Student-58 \\
\end{tabular} & 5 & 4 & 5 & 5 & 5 & 4 & 5 & 5 & 4 & 4 & 4 & 4 & 4 & 5 & 5 & 68 & 90.67 \\
\hline \begin{tabular}{|l|} 
Student-59 \\
\end{tabular} & 4 & 4 & 4 & 5 & 4 & 4 & 5 & 4 & 4 & 5 & 4 & 5 & 5 & 5 & 5 & 67 & 89.33 \\
\hline Student-60 & 5 & 4 & 4 & 5 & 5 & 4 & 4 & 5 & 5 & 5 & 5 & 4 & 5 & 4 & 5 & 69 & 92.00 \\
\hline Average & & & & & & & & & & & & & & & & & 89.01 \\
\hline
\end{tabular}

In addition to getting the results of quantitative assessment in the form of interest rating score on each instrument items by the respondents, on the results of the field trial was also obtained some suggestions for improving the features in the application. Some of the suggestions for more it completely can be seen in Table 3 . 
Table 3. Several of Suggestions which Given from Each Respondent while Conducting Field Trial

\begin{tabular}{|c|c|c|}
\hline No & Respondents & Suggestions \\
\hline 1 & Education Expert-1 & $\begin{array}{l}\text { Please, in the admin section, add the form to be able to see the } \\
\text { search for recommendations and the results of evaluation deci- } \\
\text { sions which be obtained by each evaluator. }\end{array}$ \\
\hline 2 & Informatics Expert-1 & $\begin{array}{l}\text { It needs to be added a form for managing access rights manage- } \\
\text { ment in the application. }\end{array}$ \\
\hline 3 & Education Expert-2 & $\begin{array}{l}\text { It needs to be added to the facilities to change the ad- } \\
\text { min/evaluator password. }\end{array}$ \\
\hline 4 & Informatics Expert-2 & Please add a new form to evaluate. \\
\hline 5 & Teacher-5 & $\begin{array}{l}\text { It needs to be equipped with the access rights management form } \\
\text { to enter into the application. }\end{array}$ \\
\hline 6 & Teacher-14 & $\begin{array}{l}\text { It needs to add facilities to input new assessment data from the } \\
\text { respondents as initial data in conducting evaluations. }\end{array}$ \\
\hline 7 & Teacher-30 & $\begin{array}{l}\text { It need be added a feature to be able to do the change of ad- } \\
\text { min/evaluator password. }\end{array}$ \\
\hline 8 & Student-15 & $\begin{array}{l}\text { Please add a feature to determine who has the right to access or } \\
\text { input data into the application. }\end{array}$ \\
\hline 9 & Student-26 & $\begin{array}{l}\text { Please complete the feature to change the admin or evaluator } \\
\text { password. }\end{array}$ \\
\hline 10 & Student-41 & $\begin{array}{l}\text { Add features in the admin section to be able to see the results of } \\
\text { decisions and the results of recommendations which be obtained } \\
\text { from each evaluation process that be carried out by each evalua- } \\
\text { tor. }\end{array}$ \\
\hline 11 & Student-48 & $\begin{array}{l}\text { Add features to be able to update the password either on the } \\
\text { admin section or on the evaluator section. }\end{array}$ \\
\hline 12 & Student-56 & Add features to manage the application user access rights. \\
\hline
\end{tabular}

Stage of Revision on Field Trial Results: At this stage was revised several parts of Countenance evaluation application-oriented on ANEKA-Tri Hita Karana based on the suggestions from the respondents who had been given when on the field trial. As for the solutions that were given as a follow up based on some input/revision suggestion from the respondents it can be seen in Table 4, while that visualization of several revised parts of the application can be seen in Figure 11 to Figure 14.

Table 4. Some of the Solutions as Follow-Up From Respondents Suggestions

\begin{tabular}{|c|l|l|}
\hline No & \multicolumn{1}{|c|}{ Suggestions } & \multicolumn{1}{|c|}{ Follow-Up } \\
\hline 1 & Suggestion from education expert-1 & $\begin{array}{l}\text { Creating a recommendation data search form and the } \\
\text { results of the decisions of each evaluator in the Admin } \\
\text { section. }\end{array}$ \\
\hline 2 & Suggestion from informatics expert-1 & $\begin{array}{l}\text { Creating an access rights management form on the Admin } \\
\text { section. }\end{array}$ \\
\hline 3 & Suggestion from education expert-2 & $\begin{array}{l}\text { Creating a feature of changing the password on the admin } \\
\text { or evaluator section. }\end{array}$ \\
\hline 4 & Suggestion from informatics expert-2 & Provides features to be able to create new forms. \\
\hline 5 & Suggestion from teacher-5 & $\begin{array}{l}\text { Creating an access rights management form on the Admin } \\
\text { section. }\end{array}$ \\
\hline 6 & Suggestion from teacher-14 & Provides features to be able to create new forms. \\
\hline 7 & Suggestion from teacher-30 & Creating a password change feature in the admin or evalu- \\
\hline
\end{tabular}


Paper-Development of Countenance Application Oriented on Combining ANEKA-Tri Hita Karana as a..

\begin{tabular}{|c|l|l|}
\hline No & \multicolumn{1}{|c|}{ Suggestions } & \multicolumn{1}{|c|}{ Follow-Up } \\
\hline 8 & Suggestion from student-15 & ator section. \\
\hline 9 & Suggestion from student-26 & $\begin{array}{l}\text { Creating an access rights management form in the Admin } \\
\text { section. }\end{array}$ \\
\hline 10 & Suggestion from student-41 & $\begin{array}{l}\text { Making a password changing feature on the admin or } \\
\text { evaluator section. }\end{array}$ \\
\hline 11 & Suggestion from student-48 & $\begin{array}{l}\text { Creating a recommendation data search form and the } \\
\text { decision results of each evaluator on the Admin section. }\end{array}$ \\
\hline 12 & Suggestion from student-56 & $\begin{array}{l}\text { Creating a password changing feature on the admin and } \\
\text { evaluator section. }\end{array}$ \\
\hline
\end{tabular}

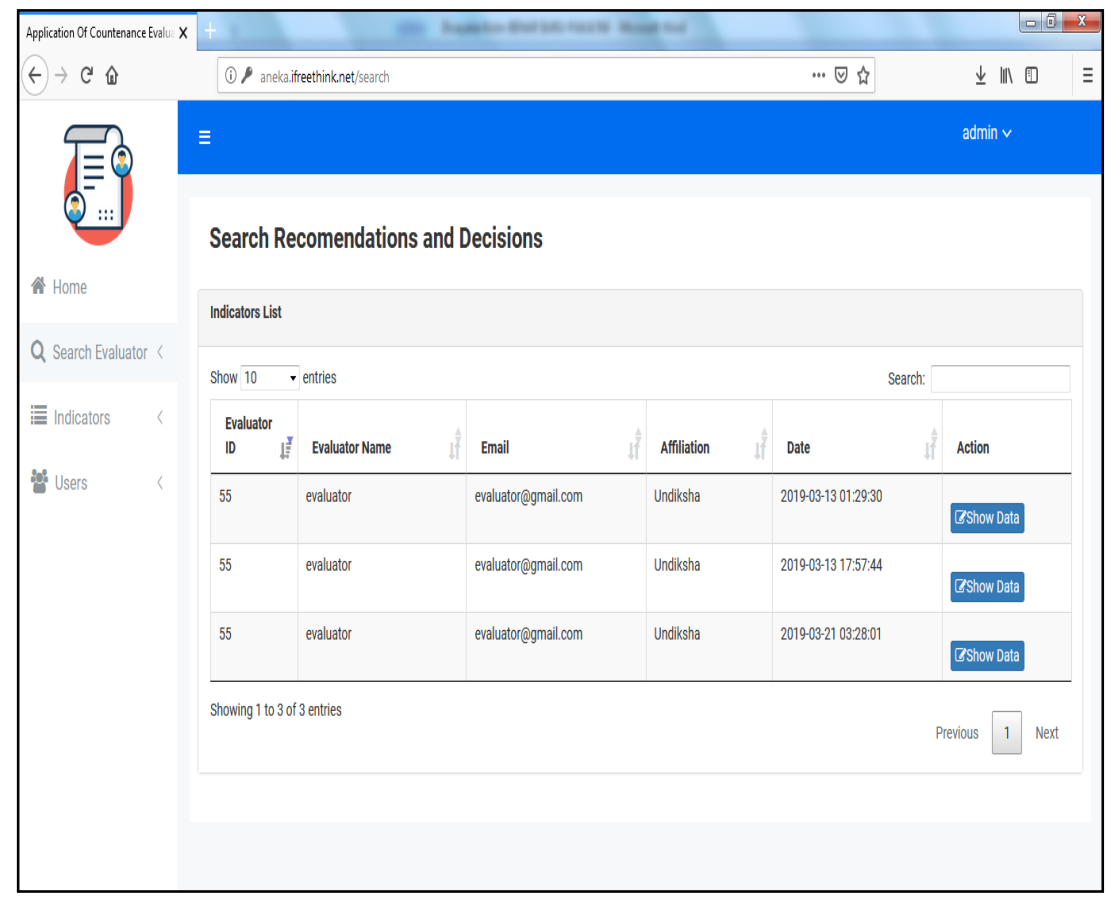

Fig. 11.Search Form of Recommendation Data and the Decision Results from Each Evaluator on the Admin Section 


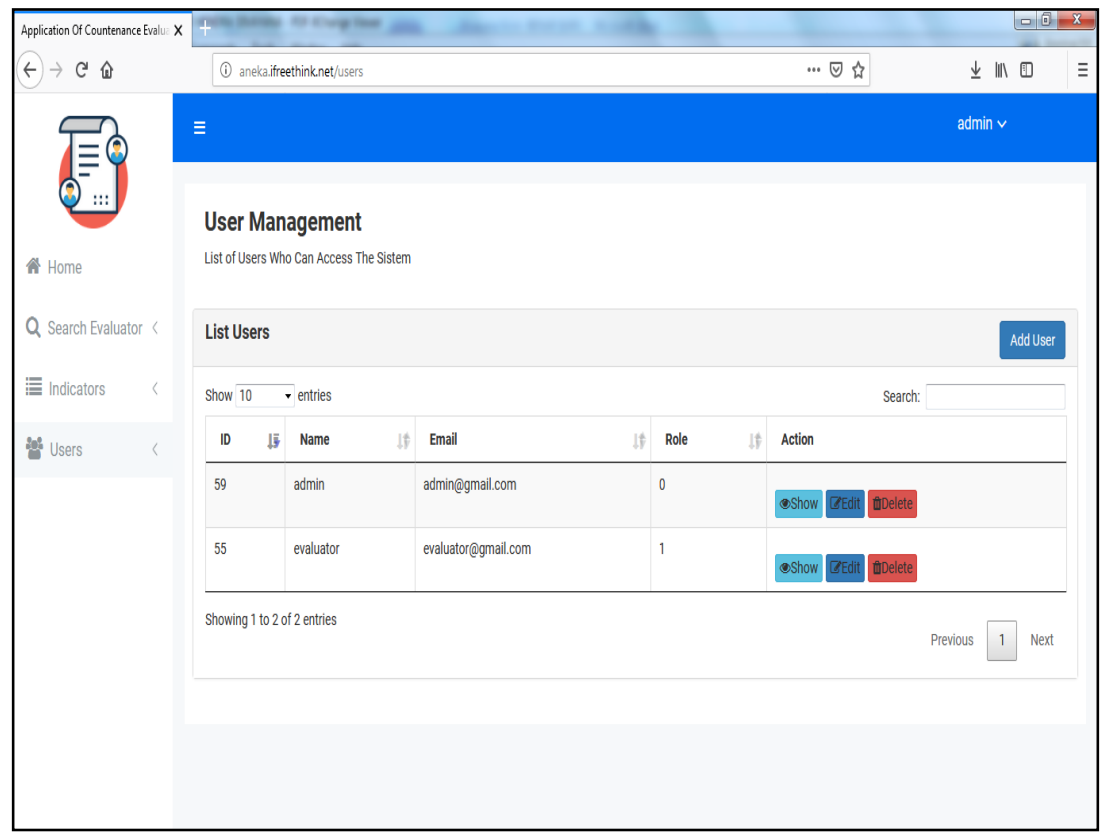

Fig. 12.The Form of Access Rights Management for Application Users

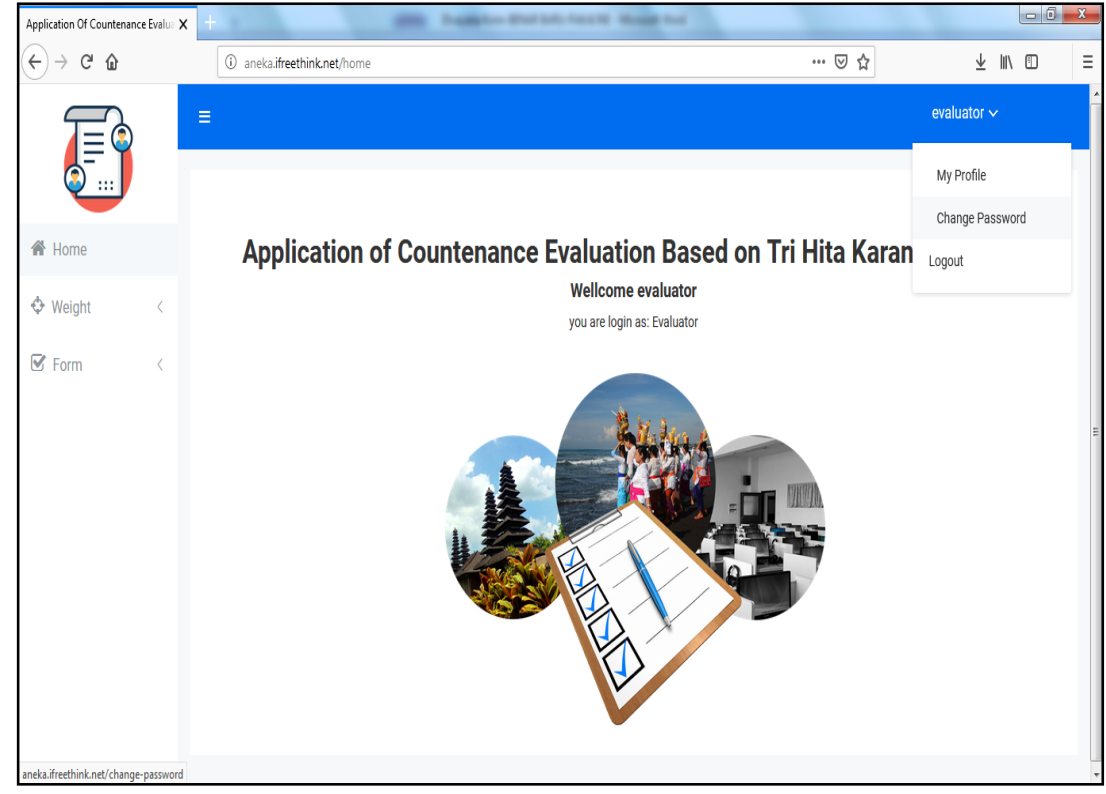

Fig. 13. The Feature for Changing Password 


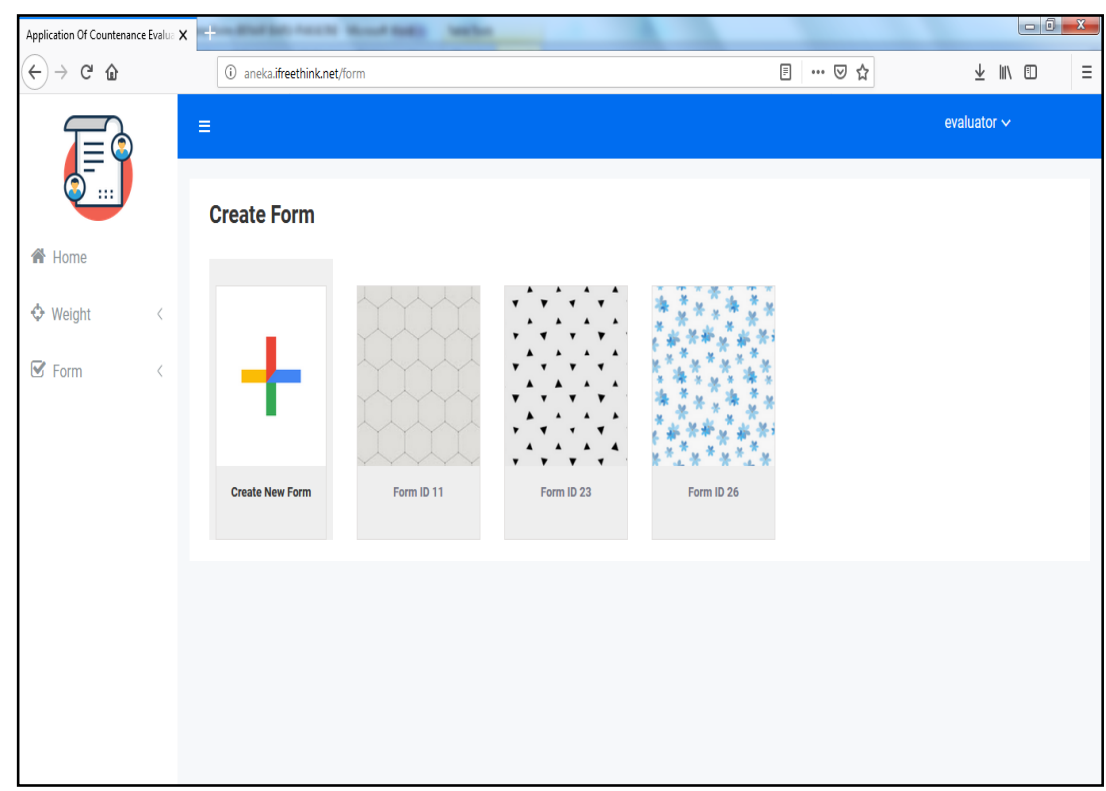

Fig. 14. The Feature for New Form Creating that Contained Student and Teacher Questionnaires

\subsection{Discussion}

Figure 1 showed the display of the login form on the Countenance evaluation application-oriented on ANEKA-Tri Hita Karana concept. The login form serves as a key for admins and evaluators to be able to enter and operate the features available in the evaluation application. Basically, this evaluation application is realized through the combination between the Countenance model, the ANEKA concept, and the Tri Hita Karana concept.

The Countenance is an evaluation model in the field of education that consists of description matrix to explain the initial conditions of the program being evaluated from the context (antecedents), process (transaction), and output (outcomes), and judgment matrix to explain the evaluation standard. This is reinforced by Gondikit's statement [14] and Dewantara's statement [15] which in principle states that the Countenance evaluation model is an evaluation model that has two components of the matrix, namely the description matrix (antecedents, transactions, and outcomes) and judgment matrix (antecedents standard, transaction standard, and outcomes standard).

$A N E K A$ is a concept that appears in Indonesia as a necessary foundation in internalizing character values and nationality in prospective civil servants in the Republic of Indonesia so that later they can realize the quality and professionalism of their work. This was strengthened by the statement of Kamal and Elim [16] which principally stated that the professionalism of prospective civil servants could be realized through the fundamental values of accountability, nationalism, public ethics, quality commitment, and anti-corruption that were internalized into their character. The word 
of ANEKA is taken from the first letters on each of the following words: Akuntabilitas, Nasionalisme, Etika Publik, Komitmen Mutu, and Anti-Korupsi (in Indonesian) or Accountability, Nationalism, Publics Ethics, Quality Commitment, and AntiCorruption (in English). It followed Hakim and Hilyana's statement [17] which states that $A N E K A$ stands for Accountability, Nationalism, Public Ethics, Quality Commitment, and Anti-Corruption.

Tri Hita Karana is a concept of Balinese local wisdom that is a cause of harmonious community life by maintaining good relationships with God (Parahyangan), good relationships with nature (Palemahan), and good relationships with humans (Pawongan). This matter is in principle the same as the statement of Astuti, Ginaya, and Susyarini [18] and the statement of Perbowosari [19] which states that Tri Hita Kara$n a$ is one of Bali's local wisdom that teaches about harmonizing life through a balance between humans and God (Parahyangan), humans with each other (Pawongan) and human with nature (Palemahan). That statement was also reinforced by the opinion of Suci et al. [20] which states that harmonization of life can occur if it can maintain good relations religiously with God (Parahyangan), socially with the community (Pawongan), and ecologically with nature (Palemahan).

Figure 2 showed the display of the main menu form on the Countenance evaluation application-oriented on ANEKA-Tri Hita Karana concept. The function of the main menu form as a navigator so can enter into all the features that were available in the application. Features that can be accessed by the admin via the main menu form, including features of recommendation search and decision results, features of indicator input, and features of user access rights management. Features that can be accessed by evaluators through the main menu form, including:

- Form input weights

- Form of antecedents on description matrix

- Form of transaction on description matrix

- Form of outcomes on description matrix

- Form of judgment matrix

- Form of recommendations and decision

- A new form for creating the feature contained questionnaires that will be filled out by students and teachers.

Figure 3 showed the appearance of indicators input form on the Countenance evaluation application-oriented on ANEKA-Tri Hita Karana concept. The indicator input form serves as a facility to enter evaluation indicator data. Data for each indicator is obtained based on derivatives of components and aspects on ANEKA integrated with the Tri Hita Karana concept.

Components and aspects of $A N E K A$ are used to measure the quality of computer knowledge that students have. ANEKA consists of five components, such as component of accountability, component of nationalism, component of public ethics, component of quality commitment, and component of anti-corruption. The component of accountability consists of several aspects, including responsibility, consistency, honesty, participation, neutrality, prioritizing the public interest, and clarity of targets. The component of nationalism consists of several aspects, including self-confidence, 
transparency, work ethic, wise, kinship, help, mutual cooperation, non-greedy, deliberation, and tolerance. The component of public ethics consists of several aspects, including respect, care, obedience to laws and regulations, high integrity, courtesy, and obedience to instructions. The component of quality commitment consists of several aspects, including effectiveness, quality-oriented, efficiency, and innovation. The component of anti-corruption consists of several aspects, including fair, caring, hard work, discipline, simple, courageous, independent.

The Tri Hita Karana aspect is used as a standard that controls the morality of IT vocational students. Tri Hita Karana consists of three aspects, such as Parahyangan, Palemahan, and Pawongan. Parahyangan aspect is used as a control standard for 'responsibility' aspect, 'consistent' aspect, and 'honest' aspect that contained in the accountability component. Parahyangan aspect is used as a control standard for 'selfconfidence' aspect, 'work' ethic aspect, 'non-greedy' aspect, 'wise' aspect, and 'transparent' aspect that contained in the nationalism component. Parahyangan aspect is used as the control standard for 'respect' aspect, 'high integrity' aspect, and 'obedient of instructions' aspect that contained in the public ethics component. Parahyangan aspect is used as a control standard for 'efficiency' aspects that contained in the quality commitment component. Parahyangan aspect is used as a control standard for 'independent' aspect, 'courageous' aspect, and 'fair' aspect that contained in the anti-corruption component. Pawongan aspect is used as a control standard for 'participation' aspects and 'prioritizing public interest' aspect that contained in the accountability component. Pawongan aspect is used as a control standard for 'help' aspect, 'tolerant' aspect, and 'deliberation' aspect that contained in the nationalism component. Pawongan aspect is used as a control standard for 'polite and meticulous' aspect that contained in the public ethics component. Pawongan aspect is used as a control standard for 'innovation' aspect and 'effectiveness' aspect that contained in the quality commitment component. Pawongan aspect is used as a control standard for 'caring' aspect that contained in the anti-corruption component. Palemahan aspect is used as a control standard for 'neutral' aspect and 'clarity of the targets' aspect that contained in the accountability component. Palemahan aspect is used as a control standard for 'mutual cooperation' aspect and 'kinship' aspects that contained in the nationalism component. Palemahan aspect is used as a control standard for 'obedience to laws and regulations' aspect that contained in the public ethics component. Palemahan aspect is used as a control standard for 'quality-oriented' aspects that contained in the quality commitment component. Palemahan aspect is used as a control standard for 'discipline' aspect, 'hard work' aspect, and 'simple' aspect that contained in the quality commitment component.

Figure 4 showed the appearance of the weight input form on the Countenance evaluation application-oriented on ANEKA-Tri Hita Karana concept. The weight input form functions as a facility to enter weight data for each evaluation aspect. The weight data for each evaluation aspect is obtained based on the average of each aspect of "ANEKA-Tri Hita Karana" divided by the total from the average "ANEKA-Tri Hita Karana." Those "ANEKA-Tri Hita Karana" aspects were formed from a combination of ANEKA components with the Tri Hita Karana concept. Figure 5 showed the form display of antecedents on description matrix at the Countenance evaluation applica- 
tion-oriented on ANEKA-Tri Hita Karana concept. The form serves to determine the average of the interest rating score, which is given by all respondents (students and teachers) in each item of the evaluation instrument. Figure 6 showed the form display of transaction on description matrix on the Countenance evaluation applicationoriented on the ANEKA-Tri Hita Karana concept. The form serves to determine the minimum value from an average of importance rating score that has been found at the antecedent stage, after that from the minimum value is obtained the code and the indicator name. Figure 7 showed the display of outcomes forms on description matrix at the Countenance evaluation application-oriented on the ANEKA-Tri Hita Karana concept. The form serves to determine priority indicators by the way to looking for the most minimum value of each minimum value that has been found at the transaction stage then from that most minimum value is obtained the code and the name of the priority indicator. Figure 8 showed the form display of judgment matrix on the Countenance evaluation application-oriented on ANEKA-Tri Hita Karana concept. The form serves to determine the indicators those are the priority of follow-up by the way to looking for normalization values and doing ranking toward the normalization value by inserting evaluation weights, so it is obtained the ranking values are sorted from lowest to highest scores. Figure 9 showed the form display of recommendations on the Countenance evaluation application-oriented on ANEKA-Tri Hita Karana concept. The form functions as a facility to show indicators that are the priority of follow-up based on the ranking results in value from the lowest to highest ranking that be obtained at the judgment matrix stage. Figure 10 shows the form display of decision on the Countenance evaluation application-oriented on ANEKA-Tri Hita Karana concept. The form serves as a facility to show one indicator that is the top priority to be improved based on the minimum value of all priority indicator lists that have been shown on the recommendation form.

Based on the results of field trials that have been presented in Table 2, showed the percentage average of evaluation application quality as much as $89.01 \%$ so that it included a very good category if it was viewed from the quality standard scores of eleven's scale that was presented in Table 1. Referring to the results of the categorization, so in general, there was no need to be done for major improvements toward the Countenance evaluation application-oriented on ANEKA-Tri Hita Karana. Even though there was no need to be done for major improvements toward the Countenance evaluation application-oriented on ANEKA-Tri Hita Karana, but from some of the inputs/suggestions given by the respondents that showed in Table 3, so it needed to do the minor revisions to improve the evaluation application.

Figure 11 showed the solution/follow-up that was carried out to make it easier for evaluation application user (from the admin side) in searching for recommendation data and looking for the results of the decisions of each evaluator. Figure 12 showed the solution/follow-up that was carried out to make it easier for the evaluation application user (from the admin side) in managing the access rights of the application users, so that it was easier to determine the granting of access rights from the admin side and the evaluator side. Figure 13 showed the solution/follow-up which was done to make it easier for users (from the admin and evaluator side) in changing the password by presenting the 'change password' feature. Figure 14 showed the solu- 
tion/follow-up which was carried out to make it easier for the user (from the evaluator side) in creating a new form that contains the evaluation instrument items which it will be filled later in by the respondents (teachers and students).

The results of this research succeeded in becoming an answer to the obstacles that were found in several previous research. The obstacle of the research that had conducted by Agung S in 2015 and also the constraints of Munteanu, Iamandi \& Tudor's research in 2015 were answered by the presence of a judgment matrix form that contained evaluation standards based on the Tri Hita Karana concept which contained the values of Balinese local wisdom, so the evaluation results can show precisely the indicators that are priority of follow-up to producing a student moral increase. The research' obstacles that were conducted by Savira and Tasrin in 2017 and the obstacles of Anggraini and Kusniarti's research in 2016 had answered by the presence of recommendation form and decision form on Countenance evaluation applicationoriented on ANEKA-Tri Hita Karana, where the form was able to show a list of priority indicators those were determined based on integrating $A N E K A$ values that had been internalized into the concept of Balinese local culture (Tri Hita Karana) so as to be able to measure cognitive, moral/affective, and even psychomotor abilities of students optimally. The research's obstacles of Toharudin and Kurniawan in 2017 had been answered by the presence of indicator input forms on Countenance evaluation application-oriented on ANEKA-Tri Hita Karana, where the indicators which were inputted into the application were obtained through the internalization results from components and aspects of $A N E K A$ that based on the Balinese local wisdom value that is Tri Hita Karana. The research obstacle of Resiki, Vipriyanti, \& Sukerta in 2018 had answered by the presence of the recommendation form on Countenance evaluation application-oriented on ANEKA-Tri Hita Karana that was able to show a list of indicators that needed follow-up started from the highest to the lowest priority.

Even though it had become the answer to the obstacles which were found in previous research, but this research is not perfect yet. This study also has several obstacles including:

- The application has not provided a feature to print the result of recommendations and decisions in the .pdf format

- The web design of application has not been responsive if used on all types of smartphone screens

\section{Conclusion}

In generally the Countenance evaluation application oriented towards integrating ANEKA values with the Tri Hita Karana concept have ready to be used to evaluate the quality of computer knowledge and student's moral at IT vocational schools on Bali Province. The evaluation application can provide recommendations and appropriate evaluation decisions so that indicators that need to get improvement priority can be immediately determined definitively and start from the highest priority to the lowest priority. The suggestions that can be given to solve the obstacles in this research include: 
- Providing a print feature of recommendations and decisions in the .pdf version

- In the future it will be better if web design of the application is developed being more responsive to all types of smartphone screens

\section{Acknowledgement}

The authors convey the deepest thanks to the Directorate of Research and Community Service, Directorate General of Strengthening Research and Development of the Ministry of Research, Technology and Higher Education of the Republic of Indonesia who had allowed conducting research and providing funding on this research through the research grant with contract number 144/UN48.16/LT/2019. The authors also did not forget to convey the thanks to Rector, Chair of the Institute of Research and Community Service of Universitas Pendidikan Ganesha and all academician of Universitas Pendidikan Ganesha who had provided moral supported for the completion of this research.

\section{References}

[1] Olaore, I.B. (2014). "The Impacts (Positive and Negative) of ICT on Education in Nigeria,” Developing Country Studies, Vol. 4., No. 23, pp. 154-156.

[2] Hernandez, R.M. (2017). "Impact of ICT on Education: Challenges and Perspectives," Propósitos y Representaciones, Vol. 5, No. 1, pp. 325-347.

[3] Ziden, A.A, Ismail, I., Spian, R., and Kumutha, K. (2011). "The Effects of ICT Use in Teaching and Learning on Students'Achievement in Science Subject in A Primary School in Malaysia," Malaysia Journal of Distance Education, Vol. 13, No. 2, pp. 19-32.

[4] Mobi, I.M., Onyenanu, I.U., and Ikwueto, O.C. (2015). "A Study of the Negative Influences of ICT on Secondary School Students in Nigeria," American Academic \& Scholarly Research Journal, Vol. 7, No. 5, pp. 136-142.

[5] Goodwin, G.P., Piazza, J., and Rozin, P. (2014). "Moral Character Predominates in Person Perception and Evaluation," Journal of Personality and Social Psychology, Vol. 106, No. 1, pp. 148-168. https://doi.org/10.1037/a0034726

[6] Agung S, L. (2015). "The Development of Local Wisdom-based Social Science Learning Model with Bengawan Solo as the Learning Source," American International Journal of Social Science, Vol. 4, No. 4, pp. 51-58.

[7] Savira, E.M., and Tasrin, K. (2017). "Involvement of Local Wisdom as A Value and An Instrument for Internalization of Public Service Innovation," International Journal of Administrative Science \& Organization, Vol. 24, No. 1, pp. 1-13. https://doi.org/10.20476/ jbb.v24i1.9464

[8] Toharudin, U., and Kurniawan, I.S. (2017). "Values of Local Wisdom: A Potential to Develop An Assessment and Remedial," International Journal of Evaluation and Research in Education (IJERE), Vol. 6, No. 1, pp. 71-78. https://doi.org/10.11591/ijere.v6i1.6349

[9] Anggraini, and Kusniarti, T. (2016).The Implementation of Character Education Model Based on Empowerment Theatre For Primary School Students," Journal of Education and Practice, Vol. 7, No. 1, pp. 26-29. 
[10] Munteanu, S.M., Iamandi, I.E., and Tudor, E. (2015). "Evaluation Model of the Entrepreneurial Character in EU Countries," Amfiteatru Economic Journal, Vol. 17, No. 38, pp. 76-92.

[11] Resiki, N.P., Vipriyanti, N.N.U., \& Sukerta, I.M. (2018). "Study of Medical Waste Management Evaluation Based on Tri Hita Karana Concept in Sanglah General Hospital, Denpasar," International Journal of Contemporary Research and Review, Vol. 9, No. 9, pp. 21050-21059. https://doi.org/10.15520/ijcrr/2018/9/09/594

[12] Ariawan, I.P.W., Simatupang, W., Ishak, A.M., Agung, A.A.G., Suratmin, Adiarta, A., and Divayana, D.G.H. (2018). "Development of ANEKA Evaluation Model Based on Topsis in Searching the Dominant Aspects of Computer Learning Quality Determinants," Journal of Theoretical and Applied Information Technology, Vol. 96, No. 19, pp. 6580-6596.

[13] Agung, A.A.G., Sudiarta, I.G.P., and Divayana, D.G.H. (2018). "The Quality Evaluation of School Management Model Based on Balinese Local Wisdom Using Weighted Product Calculation," Journal of Theoretical and Applied Information Technology, Vol. 96, No. 19 , pp. 6570-6579.

[14] Gondikit, T.J. (2018). “The Evaluation of Post PT3 Program Using Stake's Countenance Model," Malaysian Journal of Social Sciences and Humanities (MJ-SSH), Vol. 3, No. 4, pp. 109-118.

[15] Dewantara, I.P.M. (2017). "Stake Evaluation Model (Countenance Model) in Learning Process Bahasa Indonesia at Ganesha University of Educational. International Journal of Language and Literature, Vol. 1, No. 1, pp. 19-29. https://doi.org/10.23887/ijll.v1i1.9615

[16] Kamal, M., and Elim, J. (2018, September). Implementation of Project Based Learning Model for Anti Corruption Subject in Fundamental Training for BPKP's Civil Servant Candidates of the Millennials Generation. In 4th International Conference on Teacher Training and Education (ICTTE 2018), Surakarta, Indonesia. Atlantis Press, pp. 114-122. https://doi.org/10.2991/ictte-18.2018.19

[17] Hilyana, F.S., and Hakim, M.M. (2018). "Integrating Character Education on Physics Courses with Schoology-based E-Learning," Journal of Information Technology Education: Research, Vol. 17, pp. 577-593. https://doi.org/10.28945/4164

[18] Astuti, N.N.S., Ginaya, G., and Susyarini, N.P.W.A. (2019). "Designing Bali Tourism Model through the Implementation of Tri Hita Karana and Sad Kertih Values," International Journal of Linguistics, Literature and Culture, Vol. 5, No. 1, pp. 12-23. https ://doi.org/10.21744/ijllc.v5n1.461

[19] Perbowosari, H. (2019). "The Local Wisdom Value of Mandhasiya Tradition (Study of Hindu Education)," Vidyottama Sanatana International Journal of Hindu Science and Religious Studies, Vol. 3, No. 1, pp. 1-12. https://doi.org/10.25078/ijhsrs.v3i1.790

[20] Suci, I.G.S., Sonhadji K H, A., Imron, A., and Arifin, I. (2018). "Organizational Harmony in Hindu Higher Education Institution Based on Tri Hita Karana Culture," Vidyottama Sanatana International Journal of Hindu Science and Religious Studies, Vol. 2, No. 1, pp. 49-59. https://doi.org/10.25078/ijhsrs.v2i1.526

\section{$7 \quad$ Authors}

Dewa Gede Hendra Divayana is an associate professor in the field of Informatics Education at the Department of Information Technology Education, Faculty of Technical \& Vocational, Universitas Pendidikan Ganesha. His research areas are assessment and evaluation in IT education, artificial intelligence in IT education, and learning model in IT education. (email address: hendra.divayana@undiksha.ac.id). 
I Putu Wisna Ariawan is an associate professor in the field of Mathematics Education at the Department of Mathematics Education, Faculty of Mathematical \& Natural Science, Universitas Pendidikan Ganesha. His research areas are mathematics education, assessment \& evaluation in education, and statistics in education. (email address: wisna.ariawan@undiksha.ac.id).

Agus Adiarta is an associate professor in the field of Electrical Education at the Department of Electrical Education, Faculty of Technical \& Vocational, Universitas Pendidikan Ganesha. His research areas are electrical education and evaluation in education. (email address: agus.adiarta@undiksha.ac.id).

Article submitted 2019-05-14. Resubmitted 2019-07-29. Final acceptance 2019-08-08. Final version published as submitted by the authors. 